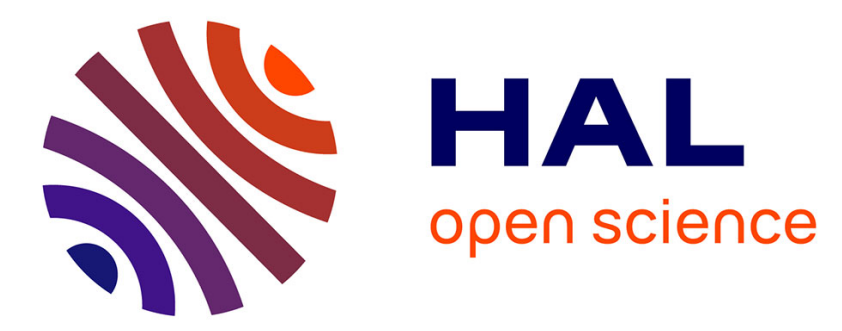

\title{
Topological Attribute Patterns for Texture Recognition
} Thanh G Nguyen, Antoine Manzanera, Walter G Kropatsch, Xuan Son

Nguyen

\section{To cite this version:}

Thanh G Nguyen, Antoine Manzanera, Walter G Kropatsch, Xuan Son Nguyen. Topological Attribute Patterns for Texture Recognition. Pattern Recognition Letters, 2016, 80, pp.91 - 97. 10.1016/j.patrec.2016.06.003 . hal-01451126

\section{HAL Id: hal-01451126 \\ https://hal.science/hal-01451126}

Submitted on 31 Jan 2017

HAL is a multi-disciplinary open access archive for the deposit and dissemination of scientific research documents, whether they are published or not. The documents may come from teaching and research institutions in France or abroad, or from public or private research centers.
L'archive ouverte pluridisciplinaire HAL, est destinée au dépôt et à la diffusion de documents scientifiques de niveau recherche, publiés ou non, émanant des établissements d'enseignement et de recherche français ou étrangers, des laboratoires publics ou privés. 


\title{
Topological Attribute Patterns for Texture Recognition
}

\author{
Thanh Phuong Nguyen ${ }^{\mathrm{a}, \mathrm{b}, \mathrm{c}, *}$, Antoine Manzanera ${ }^{\mathrm{c}}$, Walter G. Kropatsch ${ }^{\mathrm{d}}$, Xuan Son Nguyen $^{\mathrm{e}}$ \\ ${ }^{a}$ Aix Marseille Université, CNRS, ENSAM, LSIS, UMR 7296, 13397 Marseille, France \\ ${ }^{b}$ Université de Toulon, CNRS, LSIS, UMR 7296, 83957 La Garde, France \\ ${ }^{c}$ U2IS, ENSTA-Paristech, Université de Paris-Saclay, 828 bd des Maréchaux, 91762 Palaiseau Cedex, France \\ ${ }^{d}$ PRIP, TU Wien, Favoritenstr. 9/186-3, 4. Stock, A-1040 Vienna, Austria \\ ${ }^{e}$ LORIA, INRIA/CNRS/Université de Lorraine, UMR 7503, Villers-Lès-Nancy, France
}

\begin{abstract}
An efficient texture modelling framework based on Topological Attribute Patterns (TAP) is presented considering topology related attributes calculated from Local Binary Patterns (LBP). Our main contribution is to introduce new efficient mapping mechanisms that improve some typical mappings for LBP-based operators in texture classification such as rotation invariant patterns (ri), rotation invariant uniform patterns (riu2), and Local Binary Count (LBC). Like them, the proposed approach allows contrast and rotation invariant image description using more compact descriptors by projecting binary patterns to a reduced feature space. However, its expressiveness, and then its discrimination capability, is higher, since it includes additional information, related to the connected components of the binary patterns. The proposed mapping, evaluated and compared with different popular mappings, validates the interest of our approach. We then develop Complemented Patterns of Topological Attributes (CTAP) that generalise TAP model and exploit complemented information to further enhance its discrimination capability, and evaluate it on different texture datasets.
\end{abstract}

\section{Introduction}

For two decades, Local Binary Patterns (LBP) [1] have been extensively used for texture analysis, an important area of computer vision. Their decisive advantages are their low computational cost and their invariance to contrast changes, which made them attractive not only to texture recognition, but also to many other areas of computer vision.

The presented work consists of three main contributions for LBP approach. First, a family of novel mappings $T A P^{\mathcal{A}}$ is presented by considering topology-related attributes extracted from binary patterns. Second, we propose a simple yet efficient mapping $T A P^{\mathcal{A}, t}$, an improved version of the first ones, that allows to improve their discrimination power in complemented schemas while reducing the curse of dimensionality of the feature space. The two proposed mappings do not increase significantly the computational cost of basic LBPs. They extend and improve several typical mappings such as riu 2 or LBC, and are also compatible (and then can be combined) with most of the other variants. Third, we investigate the proposed mappings in complemented frameworks combining with a LBP variant to construct an efficient descriptor that is comparable to recent advances in texture classification.

The remaining of this paper is organised as follows. The next section recalls LBP works more specifically related to our work. Section 3 presents a new mapping mechanism, developped from the preliminary work [2]. Section 4 presents an

\footnotetext{
${ }^{*}$ Corresponding author

Email addresses: thanh-phuong.nguyen@univ-tln.fr (Thanh Phuong Nguyen), antoine.manzanera@ensta-paristech.fr (Antoine Manzanera), krw@prip.tuwien . ac . at (Walter G. Kropatsch), xuan-son.nguyen@inria.fr (Xuan Son Nguyen)
}

application of our mapping model to LBP variants for effective texture recognition. Section 5 is a comparative evaluation of the different descriptors derived from our models.

\section{Related works}

\subsection{General form of $L B P$}

Local Binary Patterns are introduced in a generic form in [3] as a binary code to present the local structure of a texture image by considering the centre pixel and its $P$ neighbours sampled on the centred circle of radius $R$. The sample values can be calculated by interpolation. For a scalar valued image $I$, the general form of the LBP encoding is defined as follows, for every pixel $\mathbf{p}$ :

$$
\operatorname{LBP}_{P, R}(\mathbf{p})=\sum_{i=0}^{P-1} s\left(I\left(\mathbf{q}_{i}\right)-I(\mathbf{p})\right) 2^{i},
$$

where the $\left\{\mathbf{q}_{i}\right\}$ represent the $P$ points sampled on the circle of centre $\mathbf{p}$ and radius $R$, and

$$
s(x)=\left\{\begin{array}{l}
1, x \geq 0 \\
0, \text { otherwise }
\end{array}\right.
$$

\subsection{Mapping of the LBP labels}

In practice, the basic LBP labels are not much used because of the high dimensionality of the descriptor when the number of neighbours is large. They are projected into a limiteddimensional space based on a mapping mechanism.

The circular nature of the neighbourhood justified the definition and use of local binary uniform patterns that is the most 
popular mapping of LBP labels. A LBP is said uniform when the number of bit-transitions (1-0 or 0-1) in its binary chain is at most 2. Uniform LBP based encodings (denoted $\mathrm{LBP}^{u 2}$ ) consist in discarding non uniform patterns in the global representation.

Another important notion related to the circular coding is the rotation invariant $\mathrm{LBP}$, defined as: $\mathrm{LBP}_{P, R}^{r i}=$ $\min _{0 \leq i<P}\left\{R O R\left(\operatorname{LBP}_{P, R}, i\right)\right\}$, where $\operatorname{ROR}(x, i)$ is the right circular bitwise shift of $i$ bits on the $P$-bit number $x$. The rotation invariant form of uniform patterns (denoted $\mathrm{LBP}^{\text {riu } 2}$ ), has shown impressive results for texture classification.

Zhao et al. [4] introduced Local Binary Count (LBC), inspired from [5], as an alternative mapping for LBP patterns. It discards most of the structural information of LBP by merely counting the number of $1 \mathrm{~s}$ in the binary code. Good results have been reported on rotation invariant texture classification using statistics of LBC features.

LBP mappings based on uniform patterns ignore all the geometry of non-uniform patterns that can bring important information about textural structures. Several authors have dealt with non-uniform patterns to enhance the representation power of LBP ${ }^{r i u 2}$. Liao et al. [6] and then Bianconi et al. [7] proposed to use dataset dependent dominant patterns. Nanni et al. [8] used random subspace to train features based on non-uniform patterns. Zhou et al. [9] combined non-uniform patterns by analyzing their structure and occurrence probability. Fathi and Naghsh-Nilchi [10] encoded the patterns having 4 transitions of bit (0-1 or 1-0) like riu 2 patterns by counting their number of $1 \mathrm{~s}$. The other patterns are encoded by considering their number of bit transitions.

\subsection{LBP-based variants}

The basic LBP having several limitations, such as small spatial support region, loss of local textural information, rotation and noise sensitivities, a lot of LBP variants [11] have been introduced. Different neighbourhoods, such as elliptical [12], three-patch or four-patch approaches [13] have been employed to exploit anisotropic information. In encoding step, three values $\{-1,0,1\}$ are used in Local Ternary Patterns [14] to address the issue of LBP instability on near constant image areas. Multi-structure approach [15] is considered to represent information at larger scales. Exploiting non-uniform patterns [6, 8, 9, 10, 2] is introduced to capture more useful textural information. Guo et al. [16] used a complementary component related to the magnitude of the differences. In another work, Guo et al. [17 proposed to incorporate variance as a local contrast measure into LBP histogram to take into account complementary information ignored in LBP encoding. Nguyen et al. then developed this approach by introducing Statistical Binary Patterns (SBP) [18] that explore different order moments. In [19], the discriminative patterns are selected based on a threelayered learning framework. A linear model based descriptor is introduced in [20] to take into account the microscopic configuration and local structures. Nanni et al. [21] reported a comparison for extracting features given the co-occurrence matrix using region-based approaches. In [22, 23], a more general

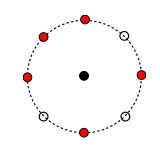

Figure 1: TAP approach

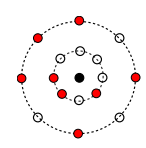

Figure 2: Multi circular supports class of LBP-based methods, namely Histograms of Equivalent Patterns, has been developed.

\section{Topological Attribute Patterns}

\subsection{Topology related attributes}

The local descriptors used by our texture model embed and generalise several rotation invariant descriptors, including uniform patterns and local binary count. They are based on a family of numerical attributes that are calculated on the original LBP. Consider the support of $\operatorname{LBP}_{P, R}$ as a set of $P$ points on a circle, where two consecutive points are said adjacent (see Figure 11). Topological information can then be extracted from the LBP using the connected components (circular runs) of $1 \mathrm{~s}$ in the pattern. We will consider the following attributes:

- Number of connected components of 1s (\#)

- Length of the largest run of 1s (M)

- Length of the smallest run of $1 \mathrm{~s}(\mathrm{~m})$

All these attributes are rotation invariant. \# is a topological measure, whose importance in the characterisation of shape is attested by a number of works in digital topology, in particular in the detection of critical points in thinning algorithms [24]. The uniform patterns correspond to $\#=1$ or 0 . $\mathrm{M}$ and $\mathrm{m}$ can be seen as extensions of the uniform pattern values to non uniform patterns. Figure 1 illustrates a non-uniform binary pattern (10111010) of 8 bits; with \# =3, M=3, $\mathrm{m}=1$.

These attributes are not independent; all configurations of values are not possible and must respect the following constraints:
1. $\mathrm{m} \leq \mathrm{M}$
2. $0 \leq \# \leq\lfloor P / 2\rfloor$
4. if \# $=1,1 \leq m=M \leq P$
3. if $\#=0, \mathrm{~m}=\mathrm{M}=0$
5. if \# $>1,1 \leq \mathrm{M} \leq P-2 \#+1$
6. if $\#>1,1 \leq \mathrm{m} \leq\lfloor P / \#\rfloor-1$

\subsection{Texture modelling}

The purpose of this work is to evaluate the contribution of the different topological attributes in texture description. The main idea is to propose a series of mappings for encoding binary patterns. First, we present $\operatorname{TAP}_{P, R}^{\mathcal{A}}$ mappings that have been firstly considered in a preliminary paper [2]. Second, we propose a new series of mappings, so called $\operatorname{TAP}_{P, R}^{\mathcal{A}, t}$, that are more efficient than the previous ones in complemented schemas.

\subsubsection{TAP $P$ P $R$ mappings}

Every version of the descriptor used in the experiments is related to a vector of $r$ topological attributes $\mathcal{A}=\left(\mathcal{A}_{1}, \ldots, \mathcal{A}_{r}\right)$ ( $1 \leq r \leq 3)$. Basically, a texture is described by computing, for each pixel $\mathbf{p}$, the LBP and its vector of $r$ attributes, denoted $\operatorname{TAP}_{P, R}^{\mathcal{A}}\left(\operatorname{LBP}_{P, R}(\mathbf{p})\right)$, and by calculating, for the whole image, 
the joint histogram of $\operatorname{TAP}_{P, R}^{\mathcal{A}}$. The total feature number of the TAP depends on $\mathrm{P}$ and the chosen subset of attributes, but it is always much smaller than $2^{P}$, the number of different LBPs (see Tab. 11. In practice, to reduce the computation time of the descriptor, we pre-compute by simple enumeration a label table $\Lambda$ which is a bijection from $\operatorname{TAP}_{P, R}^{\mathcal{A}}\left(\llbracket 0,2^{P}-1 \rrbracket\right)$ to $\llbracket 0, N_{\mathcal{A}} \rrbracket$, where $N_{\mathcal{A}}$ is the number of distinct attribute vectors. Finally we represent a texture by a histogram of labels:

$$
H(l)=\left|\left\{\mathbf{p} ; \Lambda\left(\operatorname{LBP}_{P, R}(\mathbf{p})\right)=l\right\}\right|
$$

Figure 3 shows a texture image with its corresponding label images and label histograms for the different configurations of $\operatorname{TAP}_{1,8}^{\mathcal{A}}$. In addition, Figure 4 shows images and label histograms corresponding to $\mathrm{TAP}_{1,8}^{\mathrm{AM} m}$ for different images, from the same class and from different classes. The visual (dis)similarity of histograms is apparent on the figure.

\subsection{2. $T A P_{P, R}^{\mathcal{A}, t}$ mappings}

Although smaller than traditional LBPs, the number of labels (and then of histogram bins) can become practically too big (see Tab. 1) when all three attributes $(\mathrm{M}, \mathrm{m}, \#)$ are combined, with $P=16$ or $P=24$. This will be particularly true when this mapping is applied on complemented LBP-based variant (CLBP) (see also Table 3).

To address this problem, instead of combining all three attributes, we propose to consider only two attributes ( $\mathrm{M}$ and $\mathrm{m}$ ), and to use the last one (\#) to highlight the most important patterns. Let us recall that the most popular mapping mechanisms (u2, riu2) use only uniform patterns. Fathi and Naghsh-Nilchi [10] extended the notion of uniform pattern to capture more significant patterns. These patterns have a strong link to our \# attribute, a $u 2$ pattern having $\# \leq 1$ and an extended $u 2$ pattern having $\# \leq 2$. The fact that the most significant patterns have a small value of \# suggests us using \# attribute to select patterns of $\mathcal{A}=M m$ labels. The key idea is to project all patterns having more than $t$ connected components to a unique label:

$$
\operatorname{TAP}_{P, R}^{\mathcal{A}, t}=\left\{\begin{array}{l}
0, \text { If \# }>t \\
\operatorname{TAP}_{P, R}^{\mathcal{A}}, \text { otherwise. }
\end{array}\right.
$$

Obviously the difference between feature vector sizes using $\mathrm{TAP}^{M m, t}$ and $\mathrm{TAP}_{P, R}^{M m}$ mappings is negligible since only one more bin is used if $t \geq 2$ for discarding unimportant patterns. On the other hand, $\operatorname{TAP}_{P, R}^{M m, t}$ allows to considerably reduce the feature space compared with $\mathrm{TAP}_{P, R}^{M m \#}$. In addition, by considering only the more stable patterns based on \# attribute, $\mathrm{TAP}_{P, R}^{M m, t}$ mapping may be more discriminant. In the same way, $\operatorname{TAP}_{P, R}^{M, t}$ and $\mathrm{TAP}_{P, R}^{m, t}$ can be defined to highlight the more stable patterns using the \# attribute. Because $\mathrm{TAP}_{P, R}^{M, t}, \mathrm{TAP}_{P, R}^{m, t}$ and $\mathrm{TAP}_{P, R}^{M m, t}$ simply become $\mathrm{LBP}^{\text {riu } 2}$ when $t=1$, in this work we consider only $t \geq 2$. Moreover, for $\operatorname{TAP}^{\mathcal{A}, t}$ mappings, $\mathcal{A}$ is just a subset of $\mathrm{M}, \mathrm{m}$, attribute \# is not considered anymore, then the number of $\mathrm{TAP}^{A, t}$ labels is simply equal to the number of $\mathrm{TAP}^{A}$ label, plus one, independently on the value of $t$. Table 2 presents the number of labels for $\operatorname{TAP}_{P, R}^{\mathcal{A}, t}$ mapping. It can be seen that the dimensionality of the proposed mapping is considerably reduced in comparison with the corresponding $\operatorname{TAP}_{P, R}^{\mathcal{A}}$ mapping.
Table 1: Number of different labels in Table 2: Number of different la-

\begin{tabular}{|c|c|c|c|c|c|c|c|c|c|c|c|}
\hline & \\
\hline $\mathcal{A}$ & \# & M & $\mathrm{m}$ & M\# & $\mathrm{m} \#$ & $\mathrm{Mm}$ & $\mathrm{Mm} \#$ & $\mathrm{P}$ & $\mathrm{TAP}^{M, t}$ & $\mathrm{TAP}^{m, t}$ & $\mathrm{TAP}^{M m, t}$ \\
\hline $\operatorname{TAP}_{8,1}^{A-}$ & 5 & 9 & 9 & 18 & 14 & 15 & 22 & 8 & 10 & 10 & 16 \\
\hline $\operatorname{TAP}_{16,2}^{\mathcal{A}}$ & 9 & 17 & 17 & 66 & 36 & 59 & 125 & 16 & 18 & 18 & 60 \\
\hline $\operatorname{TAP}_{24,3}^{\mathcal{H}}$ & 13 & 25 & 25 & 146 & 62 & 135 & 353 & 24 & 26 & 26 & 136 \\
\hline
\end{tabular}
$\operatorname{TAP}_{P, R}^{\mathcal{A}}$ mapping. $\quad$ bels in $\operatorname{TAP}_{P, R}^{\mathcal{A}, t}$ mappings $(t \geq 2)$.

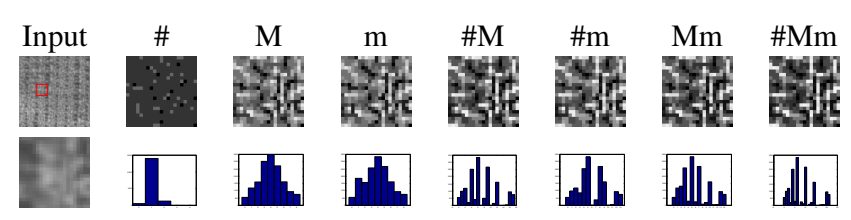

Figure 3: First row: A texture image and its label images; Second row: Zoom image, label histograms for the different configurations of attributes, with $(P, R)=(8,1)$. For the best visualization, the label images are zoomed from a part corresponding to the red square of the texture image.

\subsection{Relation with previous works}

\subsubsection{Relation with run-length texture analysis}

Our proposed mappings share a similar point with [25] since both of them exploit run-length features for texture analysis. We point out the main differences between them as follows.

- Our mapping works on a circular support of LBP, not directly on the pixel values like [25].

- In [25], a run-length is calculted in different directions and takes a value as the number of adjacent pixels having a same gray level.

- In our mapping, we consider a set of run-lengths and extract its attributes: \#, M and $\mathrm{m}$, not handled in [25].

\subsubsection{Relation with LBP-based methods}

We point out hereafter the relations between the proposed mappings and the classic mapping riu 2

- When $\operatorname{card}(\mathcal{A}) \geq 2$ and $(\# \in \mathcal{A}), \mathrm{TAP}_{P, R}^{\mathcal{A}}$ is a superset of $\mathrm{LBP}_{P, R}^{r i u 2}$ patterns. In that case indeed, riu2 patterns are distinguished, either by the value of \# and anyone among $\{\mathrm{M}, \mathrm{m}\} 11$ Therefore, for such combination of attributes $\mathcal{A}$, $\mathrm{TAP}_{P, R}^{\mathcal{A}}$ inherits the distinctive properties of $\mathrm{LBP}_{P, R}^{r i u 2}$, while containing more information. In this sense, $\operatorname{TAP}_{P, R}^{\mathcal{A}}$ generalises $\operatorname{LBP}_{P, R}^{r i u 2}$.

- When $t=1$, $\operatorname{TAP}_{P, R}^{M, t}$, $\operatorname{TAP}_{P, R}^{m, t}$ and $\operatorname{TAP}_{P, R}^{M m, t}$ are identical to $\mathrm{LBP}^{\text {riu } 2}$

- When $\operatorname{card}(\mathcal{A})=1$ or $\mathcal{A}=\{\mathrm{M}, \mathrm{m}\}, \mathcal{A}$ and riu2 are complementary.

There is a strong link between $\operatorname{TAP}_{P, R}^{\mathcal{A}, t}$ and previous works aiming at exploiting information from non-uniform patterns to improve the texture descriptors. In particular $\operatorname{TAP}_{P, R}^{\mathcal{A}, 2}$ are close to [10]. In this work, the authors extended the notion of uniform pattern to patterns having at most 4 transitions between $0-1$ and $1-0$, which corresponds to \# $=2$.

\footnotetext{
${ }^{1}$ Note that $\{\mathrm{M}, \mathrm{m}\}$ alone do not allow to distinguish uniform patterns, since the identity $\mathrm{M}=\mathrm{m}$ can occur with several connected components.
} 


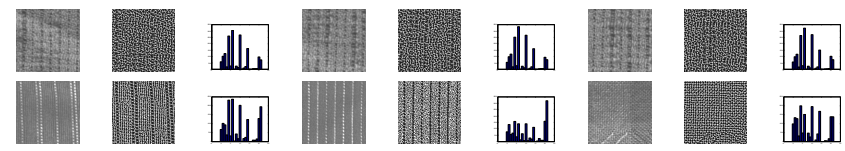

Figure 4: Texture images and their label images and histograms for $\operatorname{TAP}_{8,1}^{\# M m}$ The first row contains images of the same class, the second row contains images of different classes.

Table 3: Number of histogram bins in the complemented texture descriptors, for the different configurations.

\begin{tabular}{|l|c|c|c|c|c|c|c|}
\hline $\mathcal{A}$ & $\#$ & $\mathrm{M}$ & $\mathrm{m}$ & $\mathrm{M} \#$ & $\mathrm{~m} \#$ & $\mathrm{Mm}$ & $\mathrm{Mm} \#$ \\
\hline $\mathrm{CTAP}_{8,1}^{\mathcal{A}}$ & 50 & 162 & 162 & 648 & 392 & 450 & 968 \\
\hline $\mathrm{CTAP}_{16,2}^{\mathcal{A}}$ & 162 & 578 & 578 & 8712 & 2592 & 6962 & 31250 \\
\hline $\mathrm{CTAP}_{24,3}^{\mathcal{A}}$ & 338 & 1250 & 1250 & 42632 & 7688 & 36450 & 249218 \\
\hline
\end{tabular}

\subsection{Texture classification}

For texture classification, we use Nearest Neighbour Classifier (NNC) to evaluate our descriptor on different datasets. The $\chi^{2}$ distance between histograms is classically used to measure the similarity between two texture images [3]. If $H_{1}$ and $H_{2}$ are two attribute label histograms, the $\chi^{2}$-dissimilarity between the two textures is as follows.

$$
\chi^{2}\left(H_{1}, H_{2}\right)=\sum_{i=1}^{N_{\mathcal{A}}} \frac{\left(H_{1}(i)-H_{2}(i)\right)^{2}}{H_{1}(i)+H_{2}(i)},
$$

\section{Texture descriptor based on $\mathrm{TAP}_{P, R}^{\mathcal{A}, t}$ mappings}

We investigate our proposed mapping compared with others on LBP encoding [1] as well as on on different LBP-based variants such as Local Ternary Patterns (LTP) [14], Complemented LBP (CLBP) [16], Biological Filters (BF) [26], Statistical Binary Patterns (SBP) [18] using single or multi circular support.

\subsection{Complemented descriptor using TAP $P_{P, R}^{\mathcal{A}, t}$}

Inspired from Guo et al. [16], we developed a complemented framework for $\operatorname{TAP}_{P, R}^{\mathcal{A}}$ in the preliminary work [2]. Accordingly, the texture operator, denoted $\operatorname{CTAP}_{P, R}^{\mathcal{A}}$, is formed by the joint histograms of CTAP_S $\mathrm{S}_{P, R}^{\mathcal{A}}$ (identical to $\mathrm{TAP}_{P, R}^{\mathcal{A}}$ ), CTAP_M $\mathrm{M}_{P, R}^{\mathcal{A}}$ and CTAP_C ${ }_{P, R}$. Obviously, the number of histogram bins becomes much larger. If $N_{\mathcal{A}}$ is the dimension of $\operatorname{TAP}_{P, R}^{\mathcal{A}}$, the dimension of $\operatorname{CTAP}_{P, R}^{\mathcal{A}}$ will be $2 N_{\mathcal{A}}^{2}$. When $\operatorname{card}(\mathcal{A})>2$ and $P$ is large, the high dimension of $\operatorname{CTAP}_{P, R}^{\mathcal{A}}$ is a critical problem for the descriptor construction and the recognition performance. Table 3 displays the number of histogram bins for the different configurations. Clearly, there are many cases where the very high dimension of the descriptors prevents this method to be applied in practice, both for computational limits and for irrelevance of the descriptors, due to the sparsity of the histograms. This problem can be overcome by addressing our new operator $\operatorname{CTAP}_{P, R}^{\mathcal{A}, t}$ that will be presented hereafter.

As shown in Section 3. $\operatorname{TAP}_{P, R}^{\mathcal{A}, t}$ allows to combine more attributes without increasing significantly the dimensionality of the feature space while improving discrimination power. We remark that nothing prevents to use different mappings in
Table 4: Number of different labels in $\operatorname{CTAP}_{P, R}^{\mathcal{A}, t}$ mappings $(t \geq 2)$.

\begin{tabular}{|c|c|c|c|c|}
\hline P & CTAP $^{M, M, t}$ & CTAP $^{m, m, t}$ & CTAP $^{M m, M, t}$ & CTAP $^{M m, m, t}$ \\
\hline 8 & 200 & 200 & 320 & 320 \\
\hline 16 & 648 & 648 & 2160 & 2160 \\
\hline 24 & 1352 & 1352 & 7072 & 7072 \\
\hline
\end{tabular}

the CLBP model. On the other hand, it is well-known that the CLBP_S component is more discriminative than CLBP_M. Therefore, in order to maintain a reasonable size of descriptor, we prefer to use $\mathrm{TAP}_{P, R}^{M m, t}$ mapping to encode the first component, and $\operatorname{TAP}_{P, R}^{M, t}$ or $\operatorname{TAP}_{P, R}^{m, t}$ mapping for the second one. Let us denote " $"$ and "-" as join and concatenation operations respectively. We may for example consider the following complemented descriptor:

$$
\mathrm{CTAP}_{P, R}^{M m, M, t}=\mathrm{CLBP}_{-} S_{P, R}^{M m, t} / \mathrm{CLBP}_{-} M_{P, R}^{M, t} / \mathrm{CLBP}_{-} C_{P, R}
$$

Table 4 shows the dimensionality of $\operatorname{CTAP}^{\mathcal{A}, t}$ descriptors. Obviously, compared with Table 3 , the dimensionality is significantly reduced in complemented schemas by using $\operatorname{CTAP}^{\mathcal{A}, t}$.

\subsection{Robust texture descriptor using $C T A P_{P, R}^{\mathcal{A}, t}$}

Using the proposed mappings, we can exploit more information from non-uniform patterns. However, our mappings are also sensitive to noise since a simple change of LBP code can lead to important modifications of its attributs. In order to remedy this issue, our solution is to combine with a noise tolerant LBP-based variant. We introduce hereafter a robust texture framework for these mappings based on a recent LBPbased variant (SBP [27, 18]) to show the interest of our mapping compared to others on noisy texture images. We note that this framework can be similarly applied for other noise insensitive LBP-based variants. The principle of SBP is to separate a given texture image into different maps that are robust against noise and uniform regions in images considering different maps of moment calculated from a local support. In this paper, we address a simple version of SBP [27] based on two first order moments: mean and variance. For each filtered image, $\mathrm{CTAP}_{P, R}^{M m, M, t}$ is used to explore its texture feature. The descriptor is obtained by concatenating the feature vectors of filtered images and is denoted as SBP_CTAP $P_{P, R}^{M m, M, t}$. Moreover, TAP and CTAP exploit topological attributes considered in a single circular support. Many authors pointed out that a multi-scale approach can significantly improve the performance of LBP-based operator. In our work, M_SBP_CTAP $P_{P, R_{1}: R_{2}}^{M m, M}$ is constructed from SBP_CTAP $P_{P, R}^{M m, M, t}$ on different circular supports $(P, R)$ where $\mathrm{R}$ varies by step one from $R_{1}$ to $R_{2}$ (see Figure 2).

\section{Experiments}

\subsection{Datasets}

The effectiveness of the proposed method and the impact of the different attributes were evaluated on different representa- 
tive databases: Outex [28], CUReT [29], UIUC [30], 2D Hela [31] and KTH-TIPS 2b [32] $]^{2}$

Concerning the Outex database, we used the two popular test suites: TC10 and TC12 that are designed for texture classification under different orientations and illuminations. Two databases CUReT, UIUC use a same experimental protocol [33, 16, 30] using $N$ random images for each class to form a learning set. The classification rate is reported as the mean value together with over 100 runs. $N$ is respectively set to 46 and 20 for CUReT and UIUC datasets. The 2D Hela dataset, which consists of 862 single cell images, is decomposed into 10 classes where each class contains more than or equal to 80 images. Following [31], 80\% images of dataset are used for training and the rest is used for testing, the accuracy rate is obtained using a 5-fold cross validation. For KTH-TIPS $2 b$, we followed the training and testing scheme used in [32]. Experiments were done by training on three samples; testing is always performed on unseen samples only.

\subsection{Parameter setting}

$\operatorname{TAP}_{P, R}^{\mathcal{A}}$ was evaluated and compared with different mappings on Outex dataset in the same condition using the most commonly used single scale $(P, R)=(24,3)$.

For complemented schemas, we choose the improved version M_SBP_CTAP ${ }_{16,2: 12}^{M m, t}$ that represents a fair trade-off between discrimination power and calculation performance. In order to have a reasonable feature vector length, only 16 neighbours are considered at each scale that varies from 2 to 12 . The $\operatorname{TAP}_{P, R}^{M m, t}$ and $\mathrm{TAP}_{P, R}^{M, t}$ mappings are used respectively for encoding of CLBP_S and CLBP_M components, where $t$ is set to 2 empirically to give the best results. For SBP approach [18], the default local support $\{(1,5),(2,8)\}$ is used to calculate moments.

\subsection{Evaluation of $T A P_{P, R}^{\mathcal{A}}$ and $T A P_{P, R}^{\mathcal{A}, t}$ mappings}

The following evaluations of the proposed mapping are applied on Outex test suites using different encodings. Although it presents only the results on the configuration $(P, R)=(24,3)$, we have tested on different configurations of $(P, R)$ and the obtained conclusions are the same.

\subsubsection{LBP encoding}

Table 7 compares our proposed mapping $\left(\operatorname{TAP}_{P, R}^{\mathcal{A}}\right)$ with the different popular mappings such as $\mathrm{LBP}^{r i u 2}$ [3], $\mathrm{LBP}^{r i}$ [3], $\mathrm{LBP}^{N T}$ [10], DLBP [6] and LBC [4] on Outex dataset using LBP encoding [3]. We can make the following comments.

- The three attributes have distinct properties. Considered alone (rows 1 to 3 of Table 7), their performance is comparable to $\mathrm{LBP}_{P, R}^{r i u 2}$, except for \#, whose expressiveness is too weak if taken alone.

- Jointly considering 2 attributes (rows 4 to 6), the results are always better than $\mathrm{LBP}_{P, R}^{r i u 2}$, with an average improvement which can reach $6 \%$.

\footnotetext{
${ }^{2}$ Our code is avaiable and can be downloaded from this address: http: //tpnguyen.univ-tln.fr/download/TopoLBP
}

Table 5: Comparison between our mapping TAP ${ }^{M m, 2}$ and other mappings: riu2 and LBC considering different LBP-based encodings on Outex TC10 dataset. The configuration $(P, R)=(24,3)$ is used.

\begin{tabular}{c|ccc} 
Encoding method & TAP $^{M m, 2}$ & riu2 & LBC \\
\hline CLBP [16] & $\mathbf{9 9 . 7 6}$ & 98.93 & 98.72 \\
\hline LTP [14] & $\mathbf{9 4 . 9 2}$ & 94.77 & 90.08 \\
\hline BF [26] & $\mathbf{9 8 . 5 2}$ & 98.99 & 96.35 \\
\hline $\mathrm{SBP}_{2}[18]$ & $\mathbf{9 8 . 9 3}$ & 98.44 & 96.85
\end{tabular}

- Using all three attributes doesn't improve the results compared with a combination of two attributes. This can be explained by the fact that in this case, the number of labels is too high, which makes the histogram too sparse and reduces the effectiveness of descriptor.

- Regarding the comparison with state-of-the-art LPB mappings, our mapping $\mathrm{TAP}_{P, R}^{M m}$ is comparable with DLBP and outperforms other mappings.

- Regarding the comparison between the proposed mappings, the performances decrease from $\operatorname{TAP}_{P, R}^{\mathcal{A}}$ to $\operatorname{TAP}_{P, R}^{\mathcal{A}, t}$. This proves that the non-uniform patterns improve the discrimination power of the descriptor.

\subsubsection{LBP-based encodings}

Table 5 presents the results of our mapping on different LBPbased encodings such as: LTP, CLBP, SBP, BF compared with other mappings: LBC and $\mathrm{LBP}^{\text {riu } 2}$. It could be seen that the proposed mapping make different encoding schemes more robust than using typical mappings.

\subsection{Evaluation of proposed descriptor based on CTAP $P^{M m, M, t}$}

\subsubsection{Robust discrimination power}

This section evaluates our proposed descriptors in Section 4.2: M_SBP_CTAP ${ }_{16,2: 12}^{M m, 2}$. The dimension of the considered descriptor is $2160 \times 11=23760$. For a fair evaluation, we compare with the same frameworks but using different existing mappings: riu 2 and $L B C$. These descriptors are denoted respectively as follows: M_SBP_CLBP ${ }_{16,2: 12}^{r i u 2}$, and M_SBP_CLBC $16,2: 12$. The evaluation is carried out on different datasets: CUReT, UIUC, 2D Hela and KTH-TIPS 2b. Table 6 presents our results compared with the best results of referenced methods on these datasets.

For UIUC, CuRET and 2D Hela datasets, the results are reported by mean \pm std over 100,100 and 5 random selections respectively. Exceptfor the 2d Hela dataset, where the results are obtained using a linear SVM classifier to be comparable with other methods, the NNC is used as classifier for other datasets. In geneneral, it can be seen from this table, our proposed descriptor gives very good results on these datasets. In addition, the proposed mapping clearly contributes to the discriminative power of descriptor framework compared to other mappings: riu 2 and $\mathrm{LBC}$.

For UIUC and CuRET datasets, our descriptor clearly outperforms recent state-of-the-art results. It also gives good result on 2D Hela dataset.

For KTH-TIPS $2 \mathrm{~b}$ dataset, we compare with different methods: LBP ${ }^{\text {riu2 }}$ [3], VZ-MR8 [34], VZ-Joint [33], CLBC [4], 
CLBP [16] and NI/RD/CI [35]. As it can be seen, our framework significantly outperforms the LBP-based algorithms. Recently, Khan et al. have significantly improved the state-of-theart result $(70.6 \%)$ on KTH-TIPS $2 b$ dataset. However Khan et al. used a complex combination of many different texture descriptors: CLBP [16], WLD [36], BGP [37], LPQ [38] and BSIF [39] to improve the discrimination power. Using multiscale approach, our descriptor (M_SBP_CTAP ${ }^{M m, M, 2}$ ) still outperforms this result on KTH-TIPS $2 b$ dataset, by reaching $71.28 \%$. On the other hand, with a same texture framework, our mapping allows clearly to improve the descrimination power compared with other mappings: riu2 $(70.71 \%)$, (LBC 65.49\%). For other techniques, Cimpoi et al. [40] set a new state-of-theart result based on the combination between Improved Fisher Vector (IFV) and a deep learning technique (DeCAF).

\subsubsection{Robustness against noise}

As mentioned in Section 4.2 the proposed descriptor is robust against noise. The key idea is to take into account the extracted maps that is insensitive to noise. We consider the images from UIUC dataset by adding Gaussian noises at different levels (SNR: signal to noise ratio). Table 8 compares our descriptor with the same frameworks using different mappings: riu 2 and LBC and with other methods. Although three descriptors based on SBP are more robust against noise than two other methods thanks to noise insensitivity of SBP, the proposed descriptors is more powerful than using two mappings riu 2 and LBC.

\section{Discussion and conclusive remarks}

We have introduced a versatile and efficient framework for texture modelling and recognition, based on a family of rotation invariant attributes calculated on local binary patterns. It extends existing rotation invariant LBP based coding, including riu2 and LBC, while enhancing their expressiveness and improving their discrimination capability. Through an extensive evaluation on five recent texture datasets, the impact of the different attributes themselves has been assessed, as well as their relevance in combination with other LBP variants.

While most configurations of attributes outperform state-ofthe-art LBP based texture classification methods, several questions remain to enhance the efficiency of this framework:

- Are there other relevant rotation invariant attributes? We also experimented a fourth attribute: a dissymmetry measure, which did not improve the recognition results.

- How to combine the attributes to optimize the trade-off between recognition rate and computational performance?

- Can we extend this framework to other applications?

These questions should be addressed in future works.

${ }^{3}$ Due to the nature of $r i$ mapping that generates a huge number of labels when $P$ is large, we consider $(P, R)=(16,2)$ for $r i$ mapping.
Table 7: Comparison between the basic $\operatorname{TAP}^{\mathcal{A}}$ and different mappings such as $\mathrm{LBP}^{r i u 2}, \mathrm{LBP}^{r i}, \mathrm{LBC}, \mathrm{LBP}^{N T}$, DLBP, etc. on Outex dataset using LBP encoding.

\begin{tabular}{|c|c|c|c|c|}
\hline \multirow[t]{2}{*}{ Row number } & \multirow[t]{2}{*}{ Method } & \multicolumn{3}{|c|}{$(\mathrm{P}, \mathrm{R})=(24,3)$} \\
\hline & & TC10 & $\mathrm{TC} 12 \mathrm{t}$ & $\mathrm{TC} 12 \mathrm{~h}$ \\
\hline 1 & TAP $^{\#}$ & 76.98 & 67.50 & 58.19 \\
\hline 2 & $\mathrm{TAP}^{M}$ & 95.89 & 88.10 & 86.37 \\
\hline 3 & $\mathrm{TAP}^{m}$ & 96.48 & 86.20 & 80.37 \\
\hline$\overline{4}$ & $\overline{\text { TAP }^{M \#}}$ & 96.77 & 88.75 & 84.33 \\
\hline 5 & $\mathrm{TAP}^{m \#}$ & 97.47 & 89.28 & 85.00 \\
\hline 6 & $\mathrm{TAP}^{M m}$ & 98.12 & 92.22 & 87.20 \\
\hline$\overline{7}$ & $\mathrm{TAP}^{M m \#}$ & 97.19 & $\overline{90.93}$ & 86.71 \\
\hline$\overline{8}$ & $\mathrm{TAP}^{M, 2}$ & 95.05 & 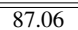 & 83.38 \\
\hline 9 & $\mathrm{TAP}^{m, 2}$ & 95.81 & 84.91 & 80.95 \\
\hline 10 & $\mathrm{TAP}^{M m, 2}$ & 96.95 & 88.56 & 85.30 \\
\hline 11 & $\mathrm{TAP}^{M, 3}$ & 95.05 & 87.06 & 84.95 \\
\hline 12 & $\mathrm{TAP}^{m, 3}$ & 96.90 & 85.83 & 80.44 \\
\hline 13 & $\mathrm{TAP}^{M m, 3}$ & 96.95 & 88.56 & 85.30 \\
\hline$\overline{14}$ & $\begin{array}{lll}\text { LBP }^{r i u 2} & 3\end{array}$ & $\begin{array}{l}94.6 \\
\end{array}$ & $\begin{array}{l}84.0 \\
\end{array}$ & 80.5 \\
\hline 15 & $\mathrm{LBP}^{N T} 10$ & 96.07 & 86.69 & 82.11 \\
\hline 16 & $\left.\mathrm{LBP}^{r i}\right]^{3}$ & 91.72 & 88.26 & 88.47 \\
\hline 17 & DLBP 6 & 98.1 & 91.6 & 87.4 \\
\hline 18 & LBC 4] & 91.22 & 83.94 & 82.34 \\
\hline
\end{tabular}

Table 8: Noise tolerance on UIUC datasets.

\begin{tabular}{c|cccc}
\multicolumn{6}{c}{ Table 8: Noise tolerance on UIUC datasets. } \\
\hline Method & SNR $=05$ & SNR $=10$ & SNR $=15$ & SNR $=30$ \\
\hline M_SBP_CTAP $_{16,2: 12}^{M m, M, 2}$ & $\mathbf{9 5 . 5 0} \pm \mathbf{0 . 9 1}$ & $\mathbf{9 6 . 2 3} \pm 0.87$ & $\mathbf{9 6 . 2 4} \pm \mathbf{0 . 7 8}$ & $\mathbf{9 6 . 3 7} \pm \mathbf{0 . 6 6}$ \\
\hline M_CLBP $_{16,2: 12}^{r i u 2}$ & $94.71 \pm 0.87$ & $95.35 \pm 0.91$ & $95.78 \pm 0.83$ & $95.66 \pm 0.65$ \\
\hline M_CLBC $_{16,2: 12}$ & $93.08 \pm 1.00$ & $93.39 \pm 0.94$ & $93.99 \pm 1.11$ & $94.57 \pm 0.65$ \\
\hline CLBP $[16]$ & 67.54 & 81.54 & 87.56 & 90.38 \\
\hline CRLBP $(\alpha=1)[47]$ & 79.20 & 88.57 & 92.74 & 93.07
\end{tabular}

\section{References}

[1] T. Ojala, M. Pietikäinen, D. Harwood, A Comparative Study of Texture Measures with Classification based on Featured Distributions, PR 29(1) (1996) 51-59.

[2] T. P. Nguyen, A. Manzanera, W. G. Kropatsch, Impact of TopologyRelated Attributes from Local Binary Patterns on Texture Classification, in: ECCV Workshops, Vol. 8926 of LNCS, 2014, pp. 80-93.

[3] T. Ojala, M. Pietikäinen, T. Mäenpää, Multiresolution Gray-scale and Rotation Invariant Texture Classification with Local Binary Patterns, PAMI 24 (2002) 971-987.

[4] Y. Zhao, D.-S. Huang, W. Jia, Completed Local Binary Count for Rotation Invariant Texture Classification, TIP 21 (10) (2012) 4492-4497.

[5] R. Zabih, J. Woodfill, Non-parametric local transforms for computing visual correspondence, in: J. Eklundh (Ed.), ECCV, Vol. 801 of LNCS, 1994, pp. 151-158.

[6] S. Liao, M. W. K. Law, A. C. S. Chung, Dominant local binary patterns for texture classification, TIP 18 (5) (2009) 1107-1118.

[7] F. Bianconi, E. González, A. Fernández, Dominant Local Binary Patterns for Texture Classification: Labelled or Unlabelled?, PRL 65 (1) (2015) $8-14$.

[8] L. Nanni, S. Brahnam, A. Lumini, A Simple Method for Improving Local Binary Patterns by considering Non-uniform Patterns, PR 45 (10) (2012) 3844-3852.

[9] H. Zhou, R. Wang, C. Wang, A Novel Extended Local Binary Pattern Operator for Texture Analysis, Inf. Sci. 178 (2008) 4314-4325.

[10] A. Fathi, A. R. Naghsh-Nilchi, Noise Tolerant Local Binary Pattern Operator for Efficient Texture Analysis, PRL 33 (9) (2012) 1093-1100.

[11] M. Pietikäinen, H. Abdenour, G. Zhao, T. Ahonen, Computer Vision Using Local Binary Patterns, 2011.

[12] S. Liao, A. C. S. Chung, Face Recognition by using Elongated Local Binary Patterns with Average Maximum Distance Gradient Magnitude, in: Y. Yagi, S. B. Kang, I.-S. Kweon, H. Zha (Eds.), ACCV, Vol. 4844 of LNCS, 2007, pp. 672-679.

[13] L. Wolf, T. Hassner, Y. Taigman, Descriptor Based Methods in The Wild, in: ECCV Workshops, 2008.

[14] X. Tan, B. Triggs, Enhanced Local Texture Feature Sets for Face Recognition Under Difficult Lighting Conditions, TIP 19 (6) (2010) 1635-1650. 


\begin{tabular}{|c|c|c|c|c|}
\hline Method & CUReT $(\mathrm{N}=46)$ & UIUC $(\mathrm{N}=20)$ & 2D Hela & KTH-TIPS $2 \mathrm{~b}\left(\mathrm{~N}_{\text {train }}=3\right)$ \\
\hline M_SBP_CTAP $_{16,2: 12}^{M m, M, 2}$ & $98.46 \pm 0.37$ & $96.41 \pm 0.89$ & $92.30 \pm 0.51$ & 71.28 \\
\hline M_SBP_CLBP $_{16,2: 12}^{r i u 2}$ & $98.21 \pm 0.36$ & $95.74 \pm 0.91$ & $91.38 \pm 1.6$ & 70.71 \\
\hline M_SBP_CLBC $_{16,2: 12}$ & $97.59 \pm 0.35$ & $94.18 \pm 1.14$ & $92.30 \pm 0.9$ & 65.49 \\
\hline SBP_CTAP $_{16,3}^{M m, M, 2}$ & $97.31 \pm 0.44$ & $95.54 \pm 0.66$ & & 69.02 \\
\hline CLBP [16] & 95.86 & 91.19 & & 67.42 \\
\hline $\mathrm{BF}+\mathrm{CLBP}[26]$ & 95.01 & 93.78 & & \\
\hline $\mathrm{DNS}+\mathrm{LBP}_{24,3}$ & 94.52 & & & \\
\hline $\mathrm{Xu}$ et al. 42 & & 92.74 & & \\
\hline BRINT2_S_M (MS9) 43 & 97.86 & & & \\
\hline Lazebnik et al. [30] & 72.5 & 96.03 & & \\
\hline EQP [44] & & & 92.0 & \\
\hline $\mathrm{EQP}_{\beta}$ & & & 92.4 & \\
\hline LTP[14] & & & 91.4 & \\
\hline DLBP $[6]$ & & & 90.0 & \\
\hline $\operatorname{dis}(\mathrm{S}+\mathrm{M})_{N, R}^{r i}[19$ & & & 95.4 & \\
\hline $\mathrm{NI} / \mathrm{RD} / \mathrm{CI}[35$ & & & & 65.0 \\
\hline VZ-Joint [33] & 98.03 & 97.83 & & 60.7 \\
\hline Khan et al. [45] & & & & 70.6 \\
\hline Timofte et al. 46 & & & & 66.3 \\
\hline DeCAF [40] & & & & 70.7 \\
\hline IFV 40 & 99.6 & 97.0 & & 69.3 \\
\hline
\end{tabular}

[15] S. Liao, X. Zhu, Z. Lei, L. Zhang, S. Z. Li, Learning Multi-scale Block Local Binary Patterns for Face Recognition, in: S.-W. Lee, S. Z. Li (Eds.), ICB, Vol. 4642 of LNCS, 2007, pp. 828-837.

[16] Z. Guo, L. Zhang, D. Zhang, A Completed Modeling of Local Binary Pattern Operator for Texture Classification, TIP 19 (6) (2010) 1657-1663.

[17] Z. Guo, L. Zhang, D. Zhang, Rotation Invariant Texture Classification using LBP Variance (LBPV) with Global Matching, PR 43(3) (2010) 706719.

[18] T. P. Nguyen, N. Vu, A. Manzanera, Statistical binary patterns for rotational invariant texture classification, Neurocomputing 173 (2016) 15651577.

[19] Y. Guo, G. Zhao, M. Pietikäinen, Discriminative features for texture description, Pattern Recognition 45 (10) (2012) 3834-3843.

[20] Y. Guo, G. Zhao, M. Pietikäinen, Texture classification using a linear configuration model based descriptor, in: BMVC, 2011, pp. 119.1-119.10.

[21] L. Nanni, S. Brahnam, S. Ghidoni, E. Menegatti, Region-based approaches and descriptors extracted from the co-occurrence matrix, IJLRST 3 (6) (2014) 192-200.

[22] F. Bianconi, A. Fernández, A unifying framework for LBP and related methods, in: Local Binary Patterns: New Variants and Applications, 2013, pp. 17-46.

[23] A. Fernández, M. X. Álvarez, F. Bianconi, Texture description through histograms of equivalent patterns, JMIV 45 (1) (2013) 76-102.

[24] S. Yokoi, J. ichiro Toriwaki, T. Fukumura, An Analysis of Topological Properties of Digitized Binary Pictures Using Local Features, Computer Graphics and Image Processing 4 (1975) 63-73.

[25] M. M. Galloway, Texture analysis using gray level run lengths, Computer graphics and image processing 4 (2) (1975) 172-179.

[26] N.-S. Vu, T. Nguyen, C. Garcia, Improving Texture Categorization with Biologically-inspired Filtering, IVC 32 (6-7) (2014) 424-436.

[27] T. P. Nguyen, A. Manzanera, Incorporating two first order moments into lbp-based operator for texture categorization, in: C. V. Jawahar, S. Shan (Eds.), ACCV Workshops, Vol. 9008 of LNCS, 2014, pp. 527-540.

[28] T. Ojala, T. Mäenpää, M. Pietikäinen, J. Viertola, J. Kyllönen, S. Huovinen, Outex - new framework for empirical evaluation of texture analysis algorithms, in: ICPR, 2002, pp. 701-706.

[29] K. J. Dana, B. van Ginneken, S. K. Nayar, J. J. Koenderink, Reflectance and Texture of Real-World Surfaces, ACM Trans. Graph. 18 (1999) 1-34.

[30] S. Lazebnik, C. Schmid, J. Ponce, A Sparse Texture Representation Using Local Affine Regions, PAMI 27 (8) (2005) 1265-1278.
[31] M. V. Boland, R. F. Murphy, A neural network classifier capable of recognizing the patterns of all major subcellular structures in fluorescence microscope images of hela cells, Bioinformatics 17 (2001) 1213-1223.

[32] B. Caputo, E. Hayman, M. Fritz, J.-O. Eklundh, Classifying Materials in the Real World, IVC 28 (1) (2010) 150-163.

[33] M. Varma, A. Zisserman, A Statistical Approach to Material Classification Using Image Patch Exemplars, PAMI 31 (11) (2009) 2032-2047.

[34] M. Varma, A. Zisserman, A Statistical Approach to Texture Classification from Single Images, IJCV 62 (1-2) (2005) 61-81.

[35] L. Liu, L. Zhao, Y. Long, G. Kuang, P. W. Fieguth, Extended local binary patterns for texture classification, IVC 30 (2) (2012) 86-99.

[36] J. Chen, S. Shan, C. He, G. Zhao, M. Pietikäinen, X. Chen, W. Gao, Wld: A robust local image descriptor, PAMI 32 (9) (2010) 1705-1720.

[37] L. Zhang, Z. Zhou, H. Li, Binary Gabor Pattern: An Efficient and Robust Descriptor for Texture Classification, in: ICIP, 2012, pp. 81-84.

[38] E. Rahtu, J. Heikkilä, V. Ojansivu, T. Ahonen, Local Phase Quantization for Blur-insensitive Image Analysis, IVC 30 (8) (2012) 501-512.

[39] J. Kannala, E. Rahtu, BSIF: Binarized Statistical Image Features, in: ICPR, 2012, pp. 1363-1366.

[40] M. Cimpoi, S. Maji, I. Kokkinos, S. Mohamed, A. Vedaldi, Describing Textures in the Wild, in: CVPR, 2014, pp. 3606-3613.

[41] F. M. Khellah, Texture Classification Using Dominant Neighborhood Structure, IEEE Trans. Image Processing 20 (11) (2011) 3270-3279.

[42] Y. Xu, H. Ji, C. Fermüller, Viewpoint Invariant Texture Description Using Fractal Analysis, IJCV 83 (1) (2009) 85-100.

[43] L. Liu, Y. Long, P. Fieguth, S. Lao, G. Zhao, BRINT: Binary Rotation Invariant and Noise Tolerant Texture Classification, TIP 23 (7) (2014) 3071-3084

[44] L. Nanni, A. Lumini, S. Brahnam, Local binary patterns variants as texture descriptors for medical image analysis, Artificial Intelligence in Medicine 49 (2) (2010) 117-125.

[45] F. S. Khan, R. M. Anwerb, J. van de Weijerc, M. Felsberga, J. Laaksonenb, Compact Color-Texture Description for Texture Classification, PRL 51 (2015) 16-22.

[46] R. Timofte, L. J. V. Gool, A Training-free Classification Framework for Textures, Writers, and Materials, in: BMVC, 2012, pp. 1-12.

[47] Y. Zhao, W. Jia, R.-X. Hu, H. Min, Completed Robust Local Binary Pattern for Texture Classification, Neurocomputing 106 (2013) 68-76. 